\title{
EVALUACIÓN Y CALIDAD DEL DESARROLLO PROFESIONAL DOCENTE, APUNTES E INTERROGANTES PARA LA UNIVERSIDAD
}

\author{
ELISA LUCARELLI*
}

Recebido: 5 mar. $2014 \quad$ Aprovado: 30 maio 2014

* Universidad Buenos Aires. Buenos Aires, Argentina. E-mail: elisalucarelli@arnet.com.ar

Resumen: La universidad es centro de inquietantes debates acerca de los procesos que desarrolla la institución en búsqueda de la excelencia en materia de calidad de la educación y, a la vez, de las articulaciones que establece con su entorno social. En la década del 90 América Latina y en particular en Argentina se instalan los mecanismos de evaluación y acreditación de carreras, y los procesos de incentivación docente en materia de investigación, sin tener en cuenta las condiciones peculiares de ese contexto. Instalar el problema de la evaluación considerando la incidencia que tienen las relaciones de poder y su circulación en la institución, posibilita analizar para qué y sobre qué indagan estos formatos evaluativos. Las investigaciones sobre el aula universitaria y las prácticas de intervención aportan a la comprensión de estos problemas.

Palabras clave: Universidad. Evaluación. Prácticas. Profesión académica.

\section{EVALUATION AND QUALITY OF TEACHER PROFESSIONAL DEVELOPMENT, NOTES AND QUESTIONS FOR UNIVERSITY}

Abstract: The University is center of disquieting debates about processes that develops the institution in search of excellence in terms of quality of education and, at the same time, joint sets with their social environment. The problems that articulates the evaluation and development professional teaching at the University is installed means consider practices that take place in the framework of the conditions back as they occur. What are the characteristics of the academic profession, who are these researchers teachers who are faced with new forms of evaluation of institutional programs and of their own practices, are some of the questions that allow you to open this problem.

Key words: Universidad. Evaluacion academic. Practicas. Profesion.

\section{AVALIAÇÃO E QUALIDADE DO DESENVOLVIMENTO PROFISSIONAL DO PROFESSOR. NOTAS E QUESTÕES PARA A UNIVERSIDADE}

Resumo: A universidade é centro de inquietantes debates sobre os processos que a instituição desenvolve em busca da excelência em matéria de qualidade da educação e, por sua vez, das articulações que estabelece com seu entorno social. Na década de 90 América Latina e em particular a Argentina instalam os mecanismos de avaliação e acreditação de carreiras e os processos de incentivo docente em matéria de pesquisa, sem levar em conta as condições peculiares desse contexto. Instalar o problema da avaliação considerando a incidência que têm as relações de poder e sua circulação na instituição possibilita analisar para quê e sobre o quê indagam esses formatos avaliativos. As pesquisas sobre a sala de aula universitária e as práticas de intervenção conduzem à compreensão desses problemas.

Palavras-chave: Universidade. Avaliação. Práticas. Profissão acadêmica. 
El mismo título de este espacio del CONGRESO es convocante ya que nos obliga a mirar, desde el ángulo de la evaluación, una problemática como es la del desarrollo profesional docente reconocida, en estos días, como central por el más alto nivel político educativo de nuestros países. En efecto en la XXIII Conferencia Iberoamericana de Educación y IV Reunión del Consejo Asesor de las Metas 2021 que se celebró en la Ciudad de Panamá en setiembre último, los integrantes de ese Consejo Asesor (en el numeral 11) expresan a los Ministros de Educación lo siguiente

Es relevante y significativo que el tema de este informe Miradas 2013 sea el desarrollo profesional docente y mejora de la educación. El Consejo Asesor está convencido de que la cuestión docente es central para la educación. La lectura de Miradas 2013 confirma la importancia de conocer mejor las condiciones de más de 7 millones de docentes que diariamente trabajan en nuestra región.

En este encuadre, la articulación entre estos dos componentes que presenta el SIMPOSIO nos convoca a un abordaje complejo, como lo es el de todo objetos signado por relaciones, obligándonos a reflexiones que van más allá de un encuadre pedagógico, si es que este se entiende solamente en su faz técnica. No es esta nuestra intención, sino que intentaremos comprender y comprehender esta relación desde los procesos y sujetos involucrados, en un contexto peculiar como es el universitario.

La universidad es, en la actualidad, centro de inquietantes debates acerca de los procesos que desarrolla la institución en búsqueda de la excelencia en materia de calidad de la educación y, a la vez, de las articulaciones que establece con su entorno social; son especialmente relevantes los debates que se generan en torno a las posibilidades que tiene la institución para poder identificar y atender a las demandas que la acucian, demandas que manifiestan las ambigüedades y contradicciones propias de un mundo en transición, en el que los cambios tecnológicos y de la información no se constituyen ,de por sí, en fuentes de distribución más equitativa de bienes entre las poblaciones. La universidad, en una sociedad democrática, debería ser el ámbito favorecedor de la construcción de un pensamiento alternativo que diera lugar a la consolidación de espacios de resistencia y libertad intelectual necesarios para poder intervenir en ese entorno y, fundamentalmente, en su propia institución, con posiciones críticas y de autonomía personal y profesional (IMBERNON, 2000, p. 39).

Por otro lado referirnos a evaluación y desarrollo profesional docente nos abre la puerta para encara el análisis de las prácticas en la universidad lo que obliga a considerar las condiciones sociohistóricas en que esas prácticas se 
producen (EDELSTEIN, 2012). Las universidades latinoamericanos, en países asolados por dictaduras feroces en la década del 60 y del 70, asistieron a los procesos de recuperación democrática con mayores esperanzas en la ciudadanía y sus procesos de recuperación que condiciones económicas para afrontarlas. Las políticas neoliberales (o neoconservadoras) de la década de los 90 significaron achicamiento del estado, desregulación en materia de políticas públicas y a la vez énfasis en el control de los procesos de instituciones de fuerte tradición de autonomía. La función de control, que en épocas autoritarias se centraba en los aspectos político ideológicos, en el período iniciado en esa década se revierte a medidas que inciden fuertemente en lo académico administrativo, afectando a la estructura y gestión de las instituciones y a la procesos de la comunidad académica.

En efecto: El crecimiento exponencial de la matrícula, (después de periodos de restricción en el ingreso y el cierre de carreras sospechadas como atentatorias contra el orden establecido) dio lugar a cambios demográficos, cuantitativos y cualitativos, que se constituyeron en desafíos a los cuales las antiguas estructuras institucionales no siempre estuvieron en condiciones de afrontar exitosamente.

Algunos datos cuantitativos nos permiten precisar la situación. Las nuevas poblaciones ingresaron en aulas con estructuras antiguas y consolidadas, bajo la responsabilidad de docentes poco preparados para enseñar a estudiantes también nuevos en cuanto a lo que a cultura universitaria se refiere: muchos, la mayoría, primera generación de universitarios, cuyo habitus no siempre le permite dar respuestas satisfactorias a las exigencias académicas. Las universidades argentinas siguen mostrando estadísticas que revelan que sólo un 20 a $25 \%$ por cierto de quienes ingresan se gradúan. Y esta cifra es más alarmante si se considera la situación de estudiantes de los sectores menos favorecidos, los cuales constituyen solo el $5 \%$ del total de graduados de la educación superior argentina.

En este contexto, a mediados de los 90 en Argentina, al igual que en otros países de la región latinoamericana (anticipados por México y Colombia), se instalan los mecanismos de evaluación y acreditación de carreras, y los procesos de incentivación docente en cuanto a sus acciones de investigación. Al decir de Chiroleu (2013, p. 13):

se inicia un proceso más intenso de políticas activas por parte de los gobiernos tendientes [...] a ejercer su poder de regulación a través de la evaluación y acreditación de la calidad. Esta se presenta como una "recomendación" de los organismos multilaterales de crédito que 
elaboran recetarios indicativos del buen funcionamiento esperado como correlato de los préstamos otorgados.

Ante este panorama, conviene preguntarse cuáles son las condiciones de la profesión académica, quiénes son esos docentes investigadores que se enfrentan con nuevas formas de evaluación de los programas institucionales y de sus propias prácticas.

Me referiré brevemente a las características que asume la profesión académica:

La profesión académica en Argentina, en expresión de los investigadores, es caracterizada por la heterogeneidad y la expansión no planificada (MARQUINA, 2012, p. 127). "La heterogeneidad intra e interinstitucional, las sucesivas rupturas institucionales e intervenciones del poder político en la vida universitaria y la expansión no planificada del cuerpo docente como producto de la ampliación de la matrícula".

Nos encontramos entonces con un cuerpo docente que se duplicó en 10 años (1982 a 1992), la mayoría con dedicaciones docentes simples y en muchos casos ad honorem, que implican 10 horas de trabajo semanal, con obligación de tarea docente, pero sin su correlato en investigación. A la vez es muy baja la cantidad de profesores con dedicación exclusiva. Esta condición salarial la diferencia negativamente la situación de la casi totalidad de instituciones de América Latina, del Norte, y europea. Las cifras nos indican en el 2006 de $14,53 \%$ de docentes de universidades nacionales con dedicación exclusiva, $24,42 \%$ con semidedicación, y $61,05 \%$ con dedicación simple. La caracterización de "esta mayoría de dedicación simple a la tarea académica pone en cuestión el concepto mismo de "profesión académica" para el caso argentino. El ingreso y promoción se hace por concurso que otorga la estabilidad por un período de tiempo, ya que, a diferencia de los países centrales, en Argentina no existe la "definitividad" en el cargo (o tenure). Mecanismo este del concurso legítimo y valedero como instancia de evaluación de la calidad docente, pero a la vez complejo para llevarse a cabo en tiempos razonablemente breves en las megauniversidades como la UBA.

Otra característica es el bajo nivel de salarios en comparación con otros países de la región (si bien se han incrementado en los últimos 5 años), situación esta que, unida a las bajas dedicaciones que comprenden al casi dos tercios de la docencia, hace que muchas universidades, sobre todo las más masivas y tradicionales, funcionen por "donación de trabajo" (MARQUINA, 2012, p. 131). En estas últimas, además, hay gran cantidad de docentes ad honorem, cerca del 
$30 \%$, básicamente graduados recientes. Sin embargo, en estas condiciones, en las universidades nacionales se produce la mayor producción científica dado que los dos tercios los artículos publicados en el país son producidos en estas instituciones. En cuanto a la formación de posgrado, la explosión de este tipo de oferta es un fenómeno reciente en Argentina, sólo un 30\% de los titulados son doctores.

Dentro de este nivel, el cuaternario, es todavía más reciente (no llega a dos décadas) la oferta de posgrados centrados en la problemática del docente universitario y, por tanto, que incluyan los aspectos políticos, administrativos, pedagógicos y didácticos de esa formación.

Me parece necesario recordar la peculiaridad de la profesión docente en la universidad, en relación a su formación y a la titulación de base. A diferencia de otros niveles del sistema, la universidad permite el ingreso y el desarrollo de la carrera docente por parte de profesionales que son valorados exclusivamente en función de la titulación profesional propia de un área disciplinar profesional determinada. Esta situación pone en relieve la propia cultura de una institución que pone en el centro de sus valores el conocimiento, su producción a través de la investigación y su transmisión a través de la enseñanza.

¿Cómo se instala en esta situación la preocupación por la evaluación y el desarrollo profesional docente? ¿Cuáles son los dispositivos que pone y que puede poner en acción la institución para el desarrollo de la profesión docente de manera de que contribuye a una mayor retención de los estudiantes?

Parece necesario abordar conceptualmente algunas cuestiones relativas a la evaluación y también acerca de la calidad en la docencia universitaria.

A la vez a pesar de que esto puede ser reiterativo en un Congreso dedicado a la evaluación, me parece importante recordar que la evaluación siempre supone comparar, contrastar una realidad particular con aquello que se considere bueno o mejor, de manera tal de emitir un juicio de valor . Según Jacques Ardoino "Evaluar consiste en juzgar colocándose desde un punto de vista, poniendo en juego uno o varios valores, estimando o apreciando, un estado, un objeto, una acción, comparando entre ellos algunos elementos valorados de manera espontánea" (2002, p. 28).

Ardoino también nos recuerda que cada acto evaluador se asienta sobre una base de prácticas evaluativas espontáneas, ancladas culturalmente, aprendidas desde la infancia a través de la experiencia vivida. Así entendido, cada acto evaluador moviliza lo racional y conciente pero también sensaciones, percepciones, emociones, afectos, implicaciones, sentimientos, representaciones, opiniones, creencias sobre aquello que se quiere apreciar. 
Por otro lado plantea que, frente a toda acción valorativa que afecte un componente educativo (en el caso de este Simposio el desarrollo profesional docente), se pueden hacer dos preguntas que, en un primer momento, es necesario disociar para entender mejor la problemática.

Una centrada en la constatación, desarrolla comparaciones que buscan la conformidad, la identidad o el alejamiento entre esto que es y aquello que debería ser. Al interrogarse acerca de en qué medida esta situación, este fenómeno observado se identifica o se aleja de la norma, el modelo, apela en su pregunta a la coherencia y a la compatibilidad dentro de un conjunto que se supone homogéneo, y se responde siguiendo una lógica hipotético deductiva. Las respuestas están así asociadas al control, dice Ardoino, pero se habla de evaluación como un término de sustitución cómoda. La otra pregunta parte de entender que la realidad analizada está constituida por hechos complejos, indisociables, heterogéneos entre ellos y por lo tanto se interroga, a través de cuestionamientos múltiples, cómo se van produciendo esos fenómenos, buscando comprender el sentido de esos procesos. Son preguntas que se inscriben en una o varias temporalidades. Se trataría de ver al desarrollo profesional docente o, mejor dicho, al desarrollo profesional de los docentes como objetos situados, como fenómenos comprensibles y evaluables en el contexto de su propia historia.

Ambas preguntas manifiestan dos formas legítimas de cuestionamiento crítico, que tienen en sí su razón de ser, pero que no expresan las mismas intencionalidades ni se refieren a la misma visión del mundo.

Roig (2013) reconoce en estas preguntas de Ardoino la presencia de dos enfoques: el de la evaluación preordenada de R. Stake y la evaluación deliberativa E. House.

Las evaluaciones preordenadas, R. Stake que se expresan en los sistemas de indicadores y se concreta a través de estándares, partiendo de una visión modélica que servirá de parámetro a la comparación. Esta visión modélica y por lo tanto los estándares son construidos por equipos de expertos, sin injerencia, por lo común, de los sujetos en evaluación.

Por su parte, las evaluaciones de tipo deliberativa E. House se fundamenta en el reconocimiento de la complejidad de los fenómenos que se dan en las instituciones educativas y de la aceptación del principio que sostiene que la sola racionalidad del conocimiento no es suficiente para movilizar procesos de cambio. Por tanto busca la comprensión de los procesos y sus contextos, destacando la importancia que tienen en esa comprensión, los procesos participativos y el análisis de la vida política de las instituciones. 
En palabras de Kemmis (KEMMIS; TARGGAT, 1988, p. 25) la evaluación sistemática se puede entender como "el proceso de obtención de información y creación de argumentos con los cuales alentar la participación de individuos y grupos en un debate crítico sobre programas específicos".

Mencionar el tema de la participación de los sujetos institucionales instala el problema de la evaluación en el contexto de las relaciones de poder y su circulación en la institución (FERNÁNDEZ, 1994); y específicamente en lo relativo a los mecanismos que diferencian la participación real de la participación simbólica en la toma de decisiones y en las distintas etapas de un proceso (SIRVENT, 1999).

Y es a partir de este punto que nos preguntamos dónde se ubica el tema de la participación en los sistemas vigentes que utilizan nuestras universidades para evaluar sus programas de formación de los estudiantes y, principalmente, en el contexto de este Simposio, para analizar el desarrollo profesional de sus docentes.

En Argentina, y a más de 20 años de debates y conflictivas resoluciones, los efectos de estas políticas de evaluación instaladas en nuestras universidades (según un consultor de UNICEF en control y evaluación citado por Roig) están impactando más en la estandarización que en ofrecer alternativas contextualizadas para instrumentar cambios o mejoras en las instituciones.

La mayoría de los procesos evaluativos del desempeño docente siguen centrados en la elaboración de cuestionarios a acerca de la efectividad de los profesores a ser contestados por los estudiantes. Dice García Garduño (2002) en una investigación sobre el tema, que este instrumento originado en las universidades estadounidenses en los años 20 del siglo pasado, (y utilizado por los $86 \%$ de esas instituciones en el año 2000) encaja dentro de la política de orientación al consumidor de ese país: si los estudiantes son los usuarios del servicio, ellos son los que deben evaluarlos. En Latinoamérica comienzan a aplicarse a fines de la década del 60, primero en las instituciones privadas para ir expandiéndose poco a poco (y no sin conflicto) a las públicas.

Es interesante plantearnos para qué y sobre qué indagan estos formatos evaluativos

El para qué se relaciona con el interés de desarrollar una enseñanza de calidad entendiendo a esta en función de la responsabilidad social que asumen las instituciones universitarias respecto de lo que hacen y generan y respecto a las personas que forman (NOSIGLIA, 2013).

En lo relativo a una de las funciones constitutivas de la Universidad como es la formación, el docente asume un rol clave, ya que se instala en un lugar 
articulador de las relaciones con la institución en su contexto social, con los estudiantes en relación al mundo del empleo, y a través de él con el capital cultural y científico de esa sociedad.

La cuestión referida a sobre qué indagan esos cuestionarios apela a la definición de los atributos del docente; al respecto son muchas las clasificaciones que hacen a la necesidad de visibilizar los aspectos que puedan orientar en el reconocimiento de los procesos a desarrollar para el mejoramiento de la calidad de la enseñanza. A título de ejemplo podemos recordar los elaborados por March (1987) y citados por Zabalza (2006, p. 182), quien realizó una síntesis de los rasgos de la enseñanza de calidad basados en cuestionarios pasados a estudiantes y en autoevaluaciones de profesores. Ellos aluden como rasgos importantes:

1) Interés y relevancia del contenido.

2) Cantidad de trabajo encomendado, incluyendo el ritmo de realización y su dificultad.

3) Organización del curso y del trabajo de cada profesor

4) Explicación (con discusión de antecedentes y consecuentes)

5) Entusiasmo, incluyendo esfuerzo y estilo

6) Apertura, estimulando la implicación del grupo

7) Empatía mostrando el interés por los estudiantes

8) Tareas, con los recursos y materiales para llevarlas a cabo

9) Procedimientos de evaluación, incluyendo la calidad del feedback.

Cabe preguntarse, ¿existe ese profesor modélico que pueda manifestar estos rasgos en sus acciones de enseñanza? ¿Es lícito plantear esos parámetros para ver en cada caso cómo se corresponde o se desvía? ¿Es necesario? ¿En qué se contribuye más a una mejor calidad de la educación y a la par al desarrollo profesional de sus docentes?

Y no será, me pregunto, que tendremos que cambiar nuestro foco de atención, cambiar la mirada y centrarla en el gusto, en el entusiasmo, en lo que los investigadores y los pensadores actuales denominan la pasión por la enseñanza? .Y recordamos sus palabras, en este caso de Christopher Day (2011, p. 191):

La enseñanza apasionada no parece afectar las normas impuestas por los órdenes social, económico y político o, incluso emocional. Sin embargo su función emancipadora consiste en influir en la capacidad de sus alumnos para entusiasmarlos con el aprendizaje, en ayudarles a elevar su mirada más allá de lo inmediato y aprender más sobre sí mismos, en construir una identidad basadas en autoimágenes nuevas. 
Y me pregunto también, cuál es la representación de la enseñanza, como práctica sinérgica de las tareas docentes, que se vislumbra detrás de estas palabras. Por cierto no es la enseñanza para el examen, "de la comercialización del terreno público del aprendizaje, de los marcos curriculares de talla única" (FRIED, 2011, p. 12).

Entiendo que esa mirada centrada en la pasión, habla de la enseñanza, en un alcance que va más allá de su dimensión técnica: implica entenderla en su multidimensionalidad, aceptando que, como práctica, conjuga realidades, dimensiones políticas, económicas, sociales, psicológicas, éticas, pedagógicas; esta mirada supone el reconocimiento de la enseñanza como práctica situada donde el sujeto que aprende, el contenido, y el contexto inmediato y amplio- el institucional, el comunitario, el del país- condicionan y marcan posibilidades. Habla de una enseñanza fundada en principios y orientada por valores. A la vez supone entender al docente como sujeto de esa práctica y no como mero ejecutor de reglamentaciones y programas externos; esta representación de su reafirmación identitaria le permite, al mismo tiempo, ver al otro sujeto de los procesos áulicos, al que aprende, como constructor de su propio aprendizaje, con sus tiempos propios, sus modalidades diversas. Reconocer de esta manera a los sujetos del aula obliga a mirar cómo enseñan y, a partir de allí, pensar en estrategias institucionales que partan del análisis de esas prácticas, para conocerlas y evaluarlas y así poder para alentar, desarrollar o mejorar esa formas de actuar en el aula.

Se trata entonces de definir formas de evaluación que coloquen a la institución universitaria en situación de pensar y de conocerse mejor a sí misma, de reconocerle a sus distintos actores la capacidad para analizar sus prácticas, revalorizándolos como sujetos en condiciones de auténtica participación. Esto es, de definir estrategias en función de reconocer cuáles son las visiones de calidad que se pueden consensuar y que orientan esas formas de actuar.

En los últimos décadas los movimientos (y la teoría) institucionalistas han alentado distintas estrategias que permiten hacer visibles desarrollos docentes que ocurren en las aulas universitarias (esto es no solamente en el salón de clases, sino en los laboratorios, clínicas, talleres, servicios) y que muchas veces son verdaderas demostraciones de innovaciones situadas. Formas protagónicas desarrolladas por los docentes para resolver los problemas con los que se enfrentan cotidianamente y que interfieren en sus propósitos de coadyuvar a la construcción de aprendizajes creativos por parte de los estudiantes. Formas alternativas a las rutinarias, que se enfrentan a las estructuras de segmentación de la teoría y la práctica, instituidas en el currículum programado y en su puesta 
en acción. Esa ruptura del statu quo implica la inclusión del estudiante como sujeto, aún cuando no se agota en las estructuras de significado subjetivo, mediante la puesta en acción de estrategias que garanticen nuevas formas de relación con el conocimiento. El estilo de enseñanza innovador, fundamentado en la tríada sujeto docente- alumno- contenido, supone modificaciones en el modelo didáctico y en su organización, de manera tal que afecten a los propósitos, los contenidos, las estrategias, los recursos, el rol que cumple el docente, rol del alumno, y, en especial, el sistema de relaciones entre estos componentes.

La experiencia innovadora supone una relación dinámica entre teoría y práctica, más allá de la simple relación de aplicación a la que esta última parece destinada en la rutina curricular o áulica.

De allí que, en oposición a la repetición, identifiquemos a la innovación, en términos de Heller, con la praxis inventiva: aquella que incluye la producción de algo nuevo en el que aprende, a través de la resolución intencional de un problema, que puede ser tanto de índole práctica como puramente teórica. En el contexto universitario, donde el predominio de lo verbal, lo disciplinar y lo conceptual en la enseñanza aparecen frecuentemente homologados con la excelencia educativa, esta visión diferente de la articulación teoría-práctica deriva en eje de innovación. Así lo hemos detectado en cátedras que centran su propuesta en el trabajo con casos, en el laboratorio, en la observación de instituciones y de sus prácticas profesionales, en la producción de objetos originales, en el análisis grupal, en el desarrollo de nuevas tecnologías, en la articulación multidisciplinaria a través del desarrollo de experiencias, situaciones todas estas donde la información teórica, los marcos conceptuales ofician de encuadre orientador y de confrontación para esas prácticas. En muchos de estos casos la práctica profesional es el elemento aglutinador para la incorporación de los problemas significativos que afectan al ejercicio profesional vigente en un campo determinado. En otros la articulación teoría-práctica se revela como estrategia metodológica en el desarrollo de toda situación de enseñanza y de aprendizaje. Estos dos sentidos que refieren a la articulación, aparecen también en nuestras investigaciones en las representaciones sociales de los sujetos protagónicos de la innovación

En este terreno nuestras investigaciones sobre el aula universitaria y las prácticas de intervención que las acompañan nos han permitido avanzar en el reconocimiento de una situación impactante: el monto de experiencias alternativas a lo tradicional, verdaderos espacios de innovación, que son gestadas y desarrolladas por el docente en su accionar cotidiano; espacios que quedan 
ocultos en el trajinar institucional y sólo se hacen visibles hacia adentro de la vida del aula (LUCARELLI, 2009).

Los procesos de evaluación que se propongan apreciar la capacidad que tienen los profesores para desarrollar, como profesionales de la docencia, experiencias de este tipo necesitan partir del reconocimiento que la evaluación no es de por sí la varita mágica que permite abrir la puerta para la resolución de los problemas de la universidad, sino que es una herramienta necesaria para la gestión universitaria, siempre que se reconozcan las limitaciones que tiene cada uno de los enfoques y de las estrategias que se derivan de ellos. Herramienta útil a partir de saber diferenciar las necesidades de información y, según esa diferenciación, organizar instrumentos diferentes y variados en función de propuestas convenientemente discutidas en el contexto institucional.

Una perspectiva situada y democrática de la evaluación obliga a consensuar las propuestas, los dispositivos a emplear y los tiempos de aplicación con una actitud de resguardo ético, de respeto por los sujetos involucrados (ROIG, 2013 , p. 28). La adopción de este encuadre de consenso técnico y político implica, desde el inicio, la puesta en acción de procesos participativos en la elaboración y desarrollo de las propuestas y que, por tanto, incluyan: el debate teórico sobre la evaluación, donde se analicen fundamentos y valores puestos en juego; la inclusión de equipos técnicos que colaboren (en una relación de acompañamiento) durante este proceso través de la coordinación, la animación de la participación, el aporte de fundamentos teóricos para la toma de decisiones, y el estímulo para un análisis crítico de la información.

Parece necesario comprender que el profesor posee un conocimiento sobre la enseñanza y que por ello es posible considerar su perspectiva como alternativa complementaria de otras formas vigentes de evaluación. Aceptar que el profesional que se dedica a la docencia crea su propio conocimiento sobre ella, permite plantearse, desde la institución y desde las políticas de evaluación, a qué se refiere ese conocimiento práctico sobre la enseñanza y desde allí definir estrategias para desarrollarla. A título de ejemplo los colegas mexicanos de la Universidad Autónoma de Aguas Calientes se preguntan, dentro de este encuadre, "por qué no diseñar un curso-taller en el que el profesor reconstruya y sistematice su conocimiento práctico y, con base a él, autoevalúe su enseñanza (y se lo evalúe agrego yo) y diseñe un plan de mejoramiento?" (FIGUEROA RUBALCAVA, 2002, p. 256). Los Talleres de Reflexión sobre experiencias alternativas, las Expocátedras, las Jornadas de Desarrollo Docente que hemos implementado con otros colegas desde la Universidad de Buenos Aires y luego compartimos con otras instituciones nacionales del país y del extranjero a lo 
largo de casi dos décadas, se orientaban en el mismo sentido (LUCARELLI, 2009).

La institución universitaria que emprende un proceso evaluativo de esta índole apuesta a las posibilidades de cambio, reconociendo los anclajes culturales y la importancia del mundo subjetivo de los actores como punto de partida para asumir actitudes de compromiso en esa transformación. Una propuesta de este tipo asume la temporalidad, acepta el tiempo lento de los procesos reflexivos y de la creatividad. Dejar tiempo para respirar, dice Christopher Day, quien toma de otro autor, Pryer estas palabras: "una comodidad y una previsibilidad excesiva son anestesiantes: no se puede caminar dormido en la experiencia pedagógica: la danza pedagógica es un proceso desenfrenado y caótico, una lucha que, a veces, es divertida y a veces dolorosa" (PRYER, en DAY, 2011, p. 120).

Tomando y parafraseando a C. Day, añado con él "No basta, por tanto, con fiarse de unas rutinas probadas y comprobadas, ya que la confianza excesiva o la maestría actual no siempre producen la evaluación óptima. Es importante dejar tiempo para respirar" (2011, p. 121)

\section{REFERENCIAS}

ARDOINO, J. Consideraciones teóricas sobre la evaluación en la educación. En: RUEDA BELTRÁN, M.; DÍAZ BARRIGA, F. Evaluación de la docencia. Perspectivas actuales. México: Paidós, 2002.

CHIROLEU, A. La Política universitaria como política pública. En: CHIROLEU, A. et al. Política universitaria en la Argentina: revisando viejos legados en busca de nuevos horizontes. Los Polvorines: UNGS, 2013.

DAY, C. Pasión por enseñar. La identidad personal y profesional del docente y sus valores. Madrid: Narcea, 2011.

DÍAZ BARRIGA, F. Evaluación de la docencia. Perspectivas actuales. México: Paidós, 2002.

EDELSTEIN, G. Un debate vigente y necesari: la formación docente en las universidades. En: LORENZATTI, M. Construcción cooperativa de políticas y estrategias de formación de docentes universitarios en la Región. Córdoba: Mercosur, 2012. 
FERNÁNDEZ, L. M. Instituciones educativas. Dinámicas institucionales en situaciones críticas. Buenos Aires: Paidós, 1994.

FIGUEROA RUBALCAVA, A. E. Una alternativa para la evaluación de la enseñanza en educación superior desde la perspectiva de los profesores. En: RUEDA BELTRÁN, M.; FRIED, R. Prólogo. En: DAY, C. Pasión por enseñar. La identidad personal y profesional del docente y sus valores. Madrid: Narcea, 2011.

GARCÍA GARDUÑO, J. M. Las dimensiones de la efectividad docente, validez y confiabilidad de los cuestionarios de evaluación de la docencia: síntesis de investigación institucional. En: RUEDA BELTRÁN, M.; DÍAZ BARRIGA, F. Evaluación de la docencia. Perspectivas actuales. México: Paidós. 2002.

IMBERNON, F. Un nuevo profesorado para una nueva universidad. Conciencia o presión? Revista Interuniversitaria de Formación del Profesorado, Zaragoza. n. 38, ago. 2000.

KEMMIS, S.; TARGGAT, R. Cómo planificar la investigación acción. Barcelona: Laertes, 1988.

LUCARELLI, E. La teoría y la práctica en la universidad: la innovación en las aulas. Buenos Aires: Miño y Dávila, 2009.

MARQUINA, M. La profesión académica en Argentina: principales características a partir de políticas recientes. En: FERNÁNDEZ LAMARRA, N.; MARQUINA, N. El futuro de la profesión académica. Desafíos para los países emergentes. Sáenz Peña: EDUN3F, 2012.

NOSIGLIA, M. C. Epílogo. Un cierre, nuevas aperturas. En: NOSIGLIA, M. C. (Comp.). La evaluación universitaria. Buenos Aires: Eudeba, 2013.

REUNIÓN DEL CONSEJO ASESOR DE LAS METAS 2021, 4., Panamá, 2013. In: CONFERENCIA IBEROAMERICANA DE EDUCACIÓN. 23. Ciudad de Panamá, Panamá, 10, 11 y 12 de septiembre de 2013.

ROIG; H. Evaluación preordenada y evaluación deliberativa: en busca de alternativas para la evaluación en la universidad. En: NOSIGLIA, M. C. (Comp.). La evaluación universitaria. Buenos Aires: Eudeba, 2013. 
SIRVENT, M. T. Cultura popular y participación social. Buenos Aires: Miño y Dávila, 1999.

ZABALZA, M. A. Competencias docentes para el profesorado universitario. Calidad y desarrollo profesional. Madrid: Narcea, 2006. 